\title{
Influences of the edge radius of curvature of tactile dots and bars on their Discriminability ${ }^{1}$
}

\author{
Wataru Toyoda ${ }^{\mathrm{a},{ }^{*}, \text { Kouki Doi }^{\mathrm{b}} \text { and Hiroshi Fujimoto }}{ }^{\mathrm{c}}$ \\ ${ }^{a}$ Graduate of Human Sciences, Waseda University, 2-579-15 Mikajima, Tokorozawa City, Saitama Prefecture, \\ 359-1192, Japan, tel. +81-4-2949-8113 ext.3625, E-mail: wataru_toyoda@toki.waseda.jp \\ ${ }^{\mathrm{b}}$ Department of Teacher Training and Information, National Institute of Special Needs Education, 5-1-1 Nobi, \\ Yokosuka City, Kanagawa Prefecture 239-8585, Japan, tel.+81-46-839-6849, E-mail: doi@nise.go.jp \\ ${ }^{\mathrm{c}}$ Faculty of Human Sciences, Waseda University, 2-579-15 Mikajima, Tokorozawa City, Saitama Prefecture, 359- \\ 1192, Japan, tel. +81-4-2949-8113 ext.3617,E-mail: fujimoto@waseda.jp
}

\begin{abstract}
Tactile dots and bars serve as tactile landmarks so that people with visual impairment can use same consumer products as those used by sighted people. However, reliable age-related data on the appropriate sizes and the shapes was not necessarily available. The purpose of this study is to evaluate influences of edge radius of curvature of tactile dots and bars on their discriminability in younger and older people to determine the appropriate size of tactile bars (as distinguished from tactile dots). Sighted younger and older participants tactually discriminated the tactile dots and bars presented individually, in random order, by a two-alternative forced-choice task. The results showed that both participants discriminated tactile bars from tactile dots faster and more accurately as the dimensional difference between bar length and width increased, regardless of conditions of edge radius of curvature. Therefore, longer dimensional difference between width and length of tactile bars is an important factor to discriminate tactile bars correctly. On the other hand, tactile dots with a larger edge radius of curvature have higher discriminability than tactile dots with a smaller edge radius of curvature in the case of dots of identical height.
\end{abstract}

Keywords: Accessibility, ISO 24503, JIS S 0011, Consumer products, Tactile perception

\section{Introduction}

Many consumer-electronics manufacturers design the keys of their products to incorporate tactile dots (dot-shaped tactile symbols) and tactile bars (barshaped tactile symbols). Tactile dots and bars serve as tactile landmarks so that older people and people with visual impairments can use the same products as those used by the visually able. The Japanese Standards Association (2000) standardized tactile dots and bars to ensure consistencies [1]. Additionally, the International Organization for Standardization (2011) also set a standard for tactile dots and bars, on the basis of the Japanese standard [2]. However, sufficient reliable age-related data on the appropriate sizes and cross-section shapes of tactile dots and bars was not necessarily available. Therefore, more quantita- tive data for optimal dimensions of tactile dots and bars is required to revise existing standards and devise new standards. In this paper, we evaluated influences of edge radius of curvature of tactile bars and dots on their discriminability, in order to determine the appropriate size of tactile bars (as distinguished from tactile dots)

\section{Method}

Sighted persons participated the experiment to eliminate any effect of tactile texture experience. 10 younger (mean age 20.3 years, $\mathrm{SD}=1.8$ years) and 10 older (mean age 63.9 years, $\mathrm{SD}=2.6$ years) sighted persons tactually discriminated the tactile dots and bars presented individually, in random order, by a

*Corresponding author. E-mail: wataru_toyoda@toki.waseda.jp 
two-alternative forced-choice task. All participants were without skin injuries or dermatosis.

Dot diameter and bar length and width were controlled. Their edge radii of curvature were also controlled (Figure $1 \&$ Table 1). 65 bar conditions were presented three times each and 13 dot conditions were presented 15 times each for a total of 390 trials, so as to eliminate order effects.

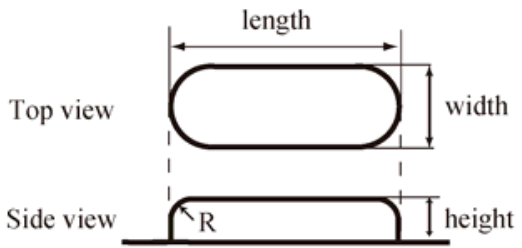

(a) Tactile bar
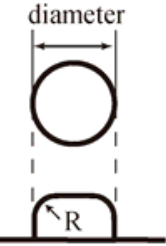

(b) Tactile dot tors of tactile dots in both participant groups for accuracy rate and discrimination time were analyzed

Table 1 Sizes of tactile bars and dots

(a) Size of tactile bars

\begin{tabular}{lc}
\hline Width $(\mathrm{mm})$ & Edge radius of curvature $(\mathrm{mm})$ \\
\hline $0.5,0.8$ & $0.0,0.25$ \\
$1.0,1.5,2.0$ & $0.0,0.25,0.5$ \\
\hline
\end{tabular}

* The actual length is the size that adds each width to each length conditions: $+0.5,+1.0,+2.0,+3.0,+4.0 \mathrm{~mm}$.

(b) Size of tactile dots

\begin{tabular}{lc}
\hline Diameter $(\mathrm{mm})$ & Edge radius of curvature $(\mathrm{mm})$ \\
\hline $0.5,0.8$ & $0.0,0.25$ \\
$1.0,1.5,2.0$ & $0.0,0.25,0.5$ \\
\hline
\end{tabular}

Fig. 1 Dimensions of tactile bar and dot used in the experiment

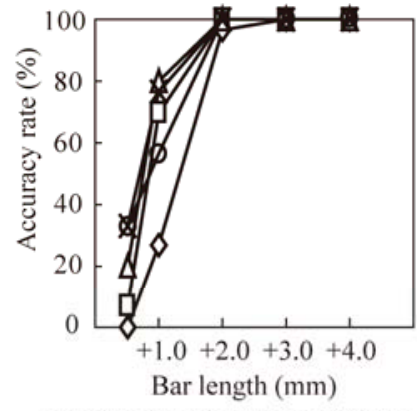

Edge radius of curvature $0.0 \mathrm{~mm}$

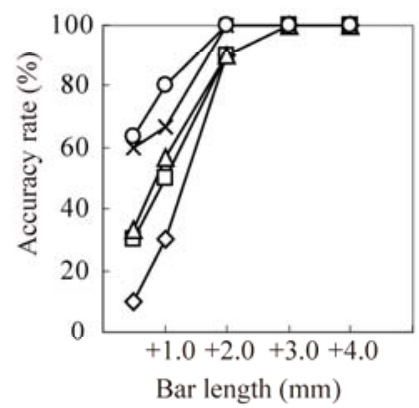

Edge radius of curvature $0.0 \mathrm{~mm}$

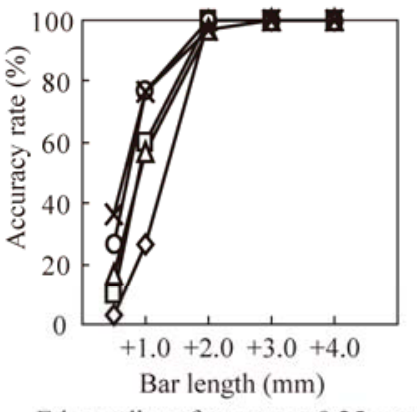

Edge radius of curvature $0.25 \mathrm{~mm}$

(a) Results of younger participants

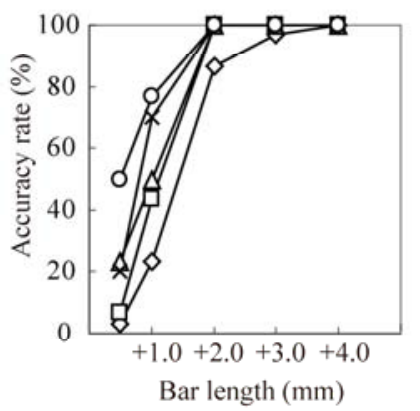

Edge radius of curvature $0.25 \mathrm{~mm}$

(b) Results of older participants

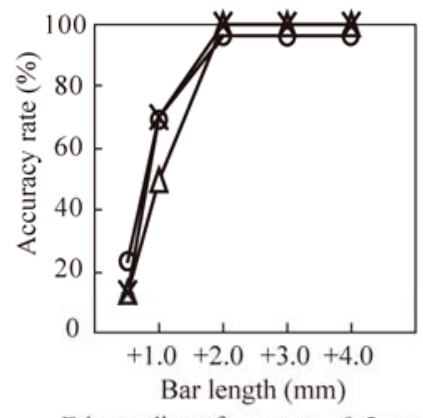

Edge radius of curvature $0.5 \mathrm{~mm}$

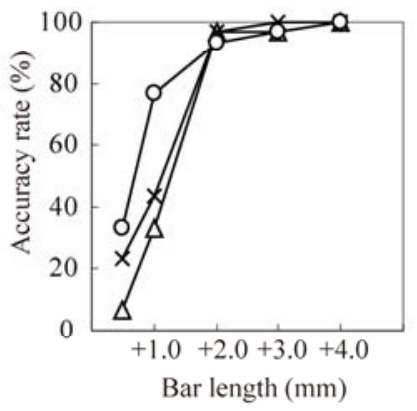

Edge radius of curvature $0.5 \mathrm{~mm}$

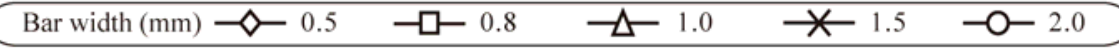

Fig. 2 Result of accuracy rate in tactile-bar conditions

Practice trials were conducted before starting the experiment. The influences of the dimensional fac- using analysis of variance (ANOVA). This study has obtained permission from the Ethical Committee on Human Research of Waseda University. 


\section{Result}

Overall, both groups discriminated tactile bars from tactile dots faster and more accurately as the dimensional difference between bar length and width increased, regardless of the conditions of width and edge radius of curvature (Figure 2). In particular, most participants in both groups distinguished tactile bars of more than $+3.0 \mathrm{~mm}$ from tactile dots in less time and with high accuracy.

On the other hand, tactile dots with a larger edge radius of curvature have higher discriminability than tactile dots with a smaller edge radius of curvature in the case of dots of identical height (Figure 3). Furthermore, both groups tended to perceive a tactile bar with a large edge radius of curvature as a tactile dot when the difference between the length and width of the bar was very small, as in the $+0.5 \mathrm{~mm}$ length condition.

\section{Discussion}

The results show that the discriminability of tactile bars depends on the difference between their length and width. Edge radius of curvature and width of the bar were not critical factors. In general, consumerelectronics manufacturers cannot necessarily control the edge radius of curvature of tactile bars, due to limitations in the manufacturing process. However, they can apply discriminable tactile bars to their products by designing them to have a sufficient difference between their width and length.

On the other hand, one possible cause of the high discriminability of tactile dots with large edge radius of curvature is a loss of their morphological characteristics. Participants might perceive tactile dots with large edge radius of curvature as mere point stimulation, because the dots do not have sharp edges easily traceable by the finger or a large, flat surface on the top. Additionally, the superficial, touchable dimensions of these tactile dots might be slightly smaller than those of dots with a small radius of curvature, because the former dots have wide skirts to which skin tissue cannot conform perfectly. Thus, participants might perceive dots with large edge radius of curvature as smaller than they really are. For these reasons, tactile dots with larger edge radius of curvature are more suitable for practical use when a tactile bar and a tactile dot are applied to a customer product concurrently.

\section{References}

[1] International Organization for Standardization, ISO/DIS 24503: Ergonomics - Accessible design - Tactile dots and bars on consumer products, International Organization for Standardization, Geneva, 2011.

[2] Japanese Standards Association, JIS S 0011: Guidelines for all people including older and people with disabilities - making tactile dots on consumer products, Japanese Standards Association, Tokyo, 2000.

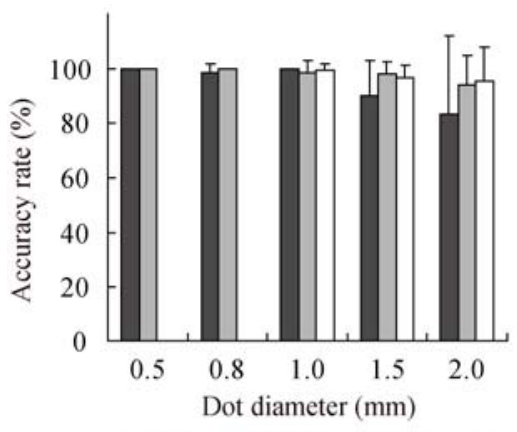

(a) Results of younger participants

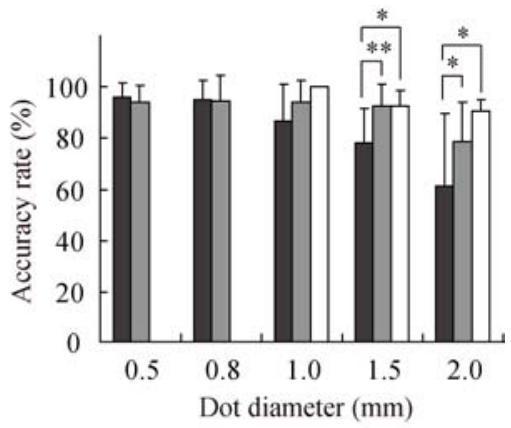

(b) Results of older participants

$$
*: p<0.05, * *: p<0.01
$$

Edge radius of curvature $(\mathrm{mm})$

0.0

$\square 0.25 \quad \square 0.5$

Fig. 3 Results of accuracy rate in tactile-dot conditions 